\title{
Impact of partial bivalent HPV vaccination on vaccine-type infection: a population-based analysis
}

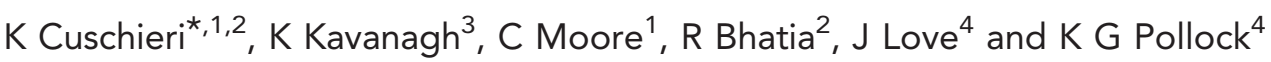 \\ ${ }^{1}$ Scottish HPV Reference Laboratory, Division of Laboratory Medicine, Royal Infirmary of Edinburgh, Edinburgh EH16 4SA, \\ Scotland; ${ }^{2}$ HPV Research Group, Queens Medical Research Institute, University of Edinburgh, Edinburgh EH16 4TJ, Scotland; \\ ${ }^{3}$ Department of Mathematics and Statistics, University of Strathclyde, 26 Richmond Street, Glasgow G1 1XH, Scotland and ${ }^{4}$ Health \\ Protection Scotland, Meridian Court, Glasgow G2 6QE, Scotland
}

Background: Data on the effectiveness of one dose of HPV vaccine are lacking, particularly in population-based settings. Data from a national HPV immunisation catch-up programme of 14-18-year-old girls were used to assess the effectiveness of $<3$ doses of the bivalent vaccine on vaccine-type and cross-reactive-type HPV infection.

Methods: Cervical samples from women attending for their first cervical smear, which had been genotyped for HPV as part of a longitudinal HPV surveillance programme were linked to immunisation records to establish the number of vaccine doses $(0,1,2$ and 3) administered. Vaccine effectiveness (VE) adjusted for deprivation and age at first dose, was assessed for prevalent HPV 16/ 18 and HPV 31/33/45 infection.

Results: VE for prevalent HPV 16/18 infection associated with 1, 2 and 3 doses was 48.2\% (95\% Cl 16.8, 68.9), 54.8\% (95\% Cl 30.7, $70.8)$ and $72.8 \%(95 \% \mathrm{Cl} 62.8,80.3)$. Equivalent VE for prevalent HPV 31/33/45 infection was $-1.62 \%(95 \% \mathrm{Cl}-85.1,45.3), 48.3 \%$ $(95 \% \mathrm{Cl} 7.6,71.8)$ and $55.2 \%(95 \% \mathrm{Cl} 32.6,70.2)$.

Conclusions: Consistent with recent aggregated trial data, we demonstrate the potential effectiveness of even one dose of HPV vaccine on vaccine-type infection. Given that these women were immunised as part of a catch-up campaign, the VE observed in this study is likely to be an underestimate of what will occur in girls vaccinated at younger ages. Further population-based studies which look at the clinical efficacy of one-dose schedules are warranted.

There is now overwhelming global evidence to demonstrate that the licensed bivalent and quadrivalent human papillomavirus (HPV) vaccines are highly efficacious for the prevention of HPV infection and associated disease when administered as a three-dose schedule. However, as described more recently, a two-dose schedule with an extended interval between doses 1 and 2 has also been demonstrated to be efficacious (Drolet et al, 2015; Lowy et al, 2015). The move to a two-dose schedule offers clear cost and logistic advantages. However, the data on the efficacy of one dose is relatively sparse. Given that the majority of countries where HPV vaccine is needed most, view national immunisation programmes as cost-prohibitive, the case and potential effectiveness for onedose schedules requires further investigation. In a recent article by Kreimer et al (2015), which used combined data from the Costa Rica Vaccine Trial and PATRICIA, the authors showed that 4 years after women aged 15-25 were vaccinated with the bivalent vaccine, one dose (and two doses administered at 0 and 1 months) provided significant protection against HPV 16/18 infection.

In Scotland, females aged 12-13 have received HPV vaccine at school since 2008 with an initial 3 year 'catch-up' for females aged 14-17 years and 364 days. Since 2011, catch-up females have entered the Scottish cervical screening programme (SCSP), which

*Correspondence: Dr K Cuschieri; E-mail: Kate.Cuschieri@luht.scot.nhs.uk

Received 15 December 2015; revised 6 March 2016; accepted 13 March 2016; published online 26 April 2016

(c) 2016 Cancer Research UK. All rights reserved 0007-0920/16 
first invites women from the age of 20. Longitudinal surveillance of vaccine impact on HPV infection and disease is possible as vaccine status-including number of doses-can be linked to a cervical screening record and a significant impact of three doses of the bivalent vaccine on both HPV infection and associated disease has been observed (Kavanagh et al, 2013, 2014; Pollock et al, 2014). Our aim in the present analysis, by supplementing our standard surveillance with additional sampling of those vaccinated with 1 and 2 doses, was to assess vaccine effectiveness (VE) on prevalent HPV infection in women who received $<3$ doses of the bivalent vaccine, thereby complementing the trial-based work of Kreimer and colleagues with a population-based analysis.

\section{MATERIALS AND METHODS}

Overview of epidemiology and surveillance programme and derivation of sample set. The overarching structure of the HPV immunisation programme in Scotland and the associated Scottish epidemiology and surveillance programme has been described elsewhere (Kavanagh et al, 2013; Kavanagh et al, 2014). In brief, one aspect of the surveillance programme involves monitoring of HPV infection in women attending for first smear. Since 2009 a yearly tranche of $\sim 2000$ residual cervical cytology samples from women (attending for first smear) has been collated from all NHS cytopathology laboratories that serve the SCSP. Collated samples were subject to HPV genotyping by the Optiplex HPV genotyping assay (Diamex, Heidelberg, Germany) which can delineate 24 HPV types including all established high-risk types. All residual samples are stored in the Scottish HPV Archive, a national repository and 'collection of collections' of cervical samples for use in research http://www.shine.mvm.ed.ac.uk/archive.shtml. Linkage of the sample through a cervical screening record to vaccination status, including number of doses was made through use of a unique identifier. The present analysis was designed specifically to report on the impact of $<3$ doses of vaccine on prevalent HPV infection and represents one of several pieces of work performed in Scotland designed to look at the impact of vaccine on various outcomes including-infection (Kavanagh et al, 2014; Bhatia et al, 2016), histological abnormalities (Pollock et al, 2014), cytology performance (Palmer et al, 2016a) and screening uptake (Palmer et al, 2016b).
Detail of sample set used for $<3$-dose analysis. Analysis was based on women who had received vaccine as part of a catch-up programme that ran for girls up to age 17 years and 364 days (women born in 1988-1993). As described above, for standard immunisation surveillance in Scotland, $\sim 2000$ samples are collated and from all laboratories that serve the SCSP (proportionate to their remit) and stored, of which $\sim 1000$ geographically representative samples are genotyped. Table 1 shows overall demographics of the population tested for the present analysis stratified by vaccine status. To augment the number of samples from women who received $<3$ doses, all additional stored samples ( $n=234)$ associated with $<3$ doses collected from women born in 1988-1993 were also tested. Thus, the final analysis incorporated a total of 300 samples from women who had received two doses, generally administered at 0 and 1 month, whereas 177 had received one dose only. This is compared to 1853 that had three doses of vaccine over the same time frame. As the unvaccinated group necessarily incorporated older birth cohorts, adjustments for birth cohort and age at receipt of vaccine were made (see below).

Analysis-vaccine effectiveness. To estimate VE, the adjusted odds ratio (OR) of prevalent infection with both HPV 16/18 and HPV 31/33/45 (in aggregate) for 1, 2 and 3 doses of vaccination were estimated using logistic regression by comparing to the unvaccinated group and VE calculated as $100^{*}(1-\mathrm{OR})$. The odds of HPV infection was adjusted for deprivation through use of the Scottish Index of Multiple Deprivation Score (SIMD), birth cohort (to account for any potential temporal changes in HPV prevalence) and age at receipt of first dose for those vaccinated, as those who were vaccinated at older ages-and therefore outside the schools based programme-were more likely to have received $<3$ doses. VE for women who received three doses over the same time frame is presented for context and comparison.

\section{RESULTS}

HPV prevalence in women with $0,1,2$ and 3 doses of vaccineunadjusted analysis. Table 2 shows the prevalence of HPV 16/18 and HPV 31/33/45 stratified according to number of vaccine doses. Prevalence of HPV 16/18 was highest in women who received 0 doses of vaccine $(29.3 \%)$ and lowest in those who received 3 doses $(11.0 \%)$-comparatively those who had one and two doses of

Table 1. Overall demographics of the population stratified by vaccine status

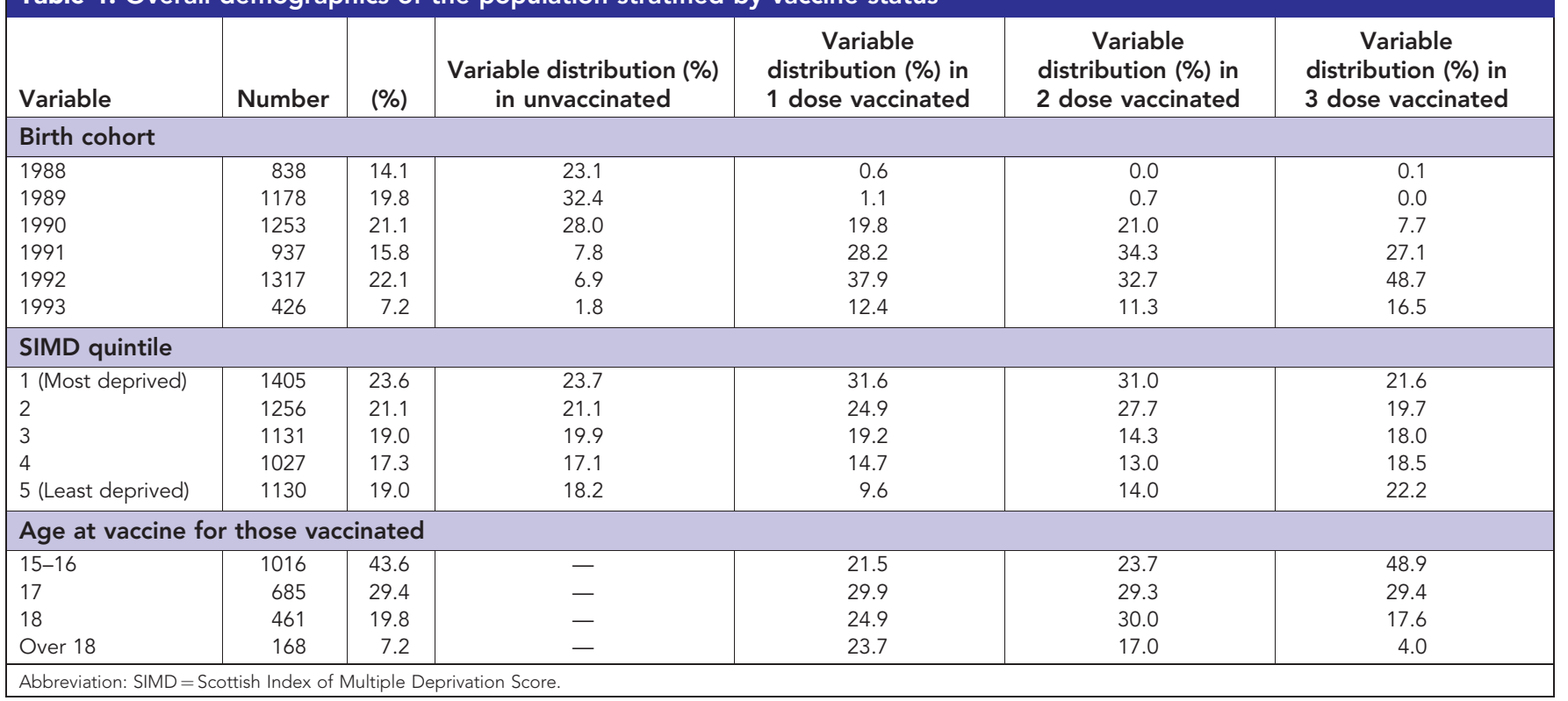


Table 2. Prevalence (unadjusted) of vaccine and crossreactive HPV types according to number of vaccine doses

\begin{tabular}{|l|c|c|c|}
\hline $\begin{array}{l}\text { No. of } \\
\text { doses }\end{array}$ & $\begin{array}{c}\text { No. of } \\
\text { samples }\end{array}$ & $\begin{array}{c}\text { HPV 16/18 } \\
\text { No. pos }(\%,(95 \% \text { Cl)) }\end{array}$ & $\begin{array}{c}\text { HPV } 31 / 33 / 45 \\
\text { No. pos }(\%,(95 \% \text { Cl) })\end{array}$ \\
\hline 0 & 3619 & $1062(29.3(27.9,30.8))$ & $468(12.9(11.9,14.1))$ \\
\hline 1 & 177 & $42(23.7(18.1,30.5))$ & $26(14.7(10.2,20.7))$ \\
\hline 2 & 300 & $63(21.0(16.8,26.0))$ & $24(8.0(5.4,11.6))$ \\
\hline 3 & 1853 & $203(11.0(9.7,12.5))$ & $115(6.2(5.2,7.4))$ \\
\hline
\end{tabular}

Abbreviations: $\mathrm{Cl}=$ confidence interval; $\mathrm{HPV}=$ human papillomavirus.

Table 3. VE of 1, 2 and 3 doses unadjusted and also adjusted for age at first vaccination, birth cohort and SIMD quintile

\begin{tabular}{|c|c|c|c|c|}
\hline $\begin{array}{l}\text { No. of } \\
\text { Doses }\end{array}$ & $\begin{array}{c}\text { Unadjusted } \\
\text { VE }(\%,(95 \% \mathrm{CI}))\end{array}$ & $P$-value & $\begin{array}{c}\text { Adjusted } \\
\text { VE }(\%,(95 \% \mathrm{Cl}))\end{array}$ & $P$-value \\
\hline \multicolumn{5}{|c|}{ HPV 16/18 } \\
\hline 1 & $25.1(-5.7,48.0)$ & 0.1093 & $48.2(16.8,68.9)$ & 0.0075 \\
\hline 2 & $36(15.3,52.3)$ & 0.0023 & $54.8(30.7,70.8)$ & $<0.0001$ \\
\hline 3 & $70.2(65.0,74.7)$ & $<0.0001$ & $72.8(63.8,80.3)$ & $<0.0001$ \\
\hline \multicolumn{5}{|c|}{ HPV $31 / 33 / 45$} \\
\hline 1 & $-15.9(-74.6,25.9)$ & 0.4978 & $-1.62(-85.1,45.3)$ & 0.9588 \\
\hline 2 & $41.4(12.1,62.8)$ & 0.0143 & $48.3(7.6,71.8)$ & 0.0287 \\
\hline 3 & $55.5(45.1,64.1)$ & $<0.0001$ & $55.2(32.6,70.2)$ & $<0.0001$ \\
\hline
\end{tabular}

vaccine had lower prevalence(s) of HPV 16/18: 23.7\% (95\% CI $18.1,30.5)$ and $21.0 \%(95 \%$ CI 16.8, 26.0), respectively, when compared to those who had 0 doses. Prevalence of HPV 31/33/45 was no different in women who received 0 or 1 dose of vaccine: $12.9 \%$ (95\% CI 11.9, 14.1) and $14.7 \%$ (95\% CI 10.2, 20.7) respectively but was lower in those who received 2 and 3 doses: $8 \%$ (95\% CI 5.4, 11.6) and 6.2\% (95\% CI 5.2, 7.4), respectively.

The unadjusted analysis showed the odds of infection with HPV $16 / 18$ in women vaccinated with 1,2 and 3 doses (compared with 0 doses) were 0.75 (95\% CI 0.52, 1.06) 0.64 (95\% CI $0.48,0.85)$ and 0.30 (95\% CI $0.25,0.35)$, respectively. Comparative odds for HPV $31 / 33 / 45$ infection were 1.16 (95\% CI $0.74,1.75), 0.59$ (95\% CI $0.37,0.88$ ), and 0.45 (95\% CI 0.36, 0.55).

VE in women with $<\mathbf{3}$ doses of vaccine. Table 3 presents VE for prevalent infection with HPV 16/18 and HPV 31/33/45, adjusted for age at first vaccination and SIMD. VE for prevalent HPV 16/18 infection associated with 1, 2 and 3 doses was $48.2 \%$ (95\% CI 16.8, 68.9), 54.8\% (95\% CI 30.7, 70.8) and 72.8\% (95\% CI 62.8, 80.3). Equivalent VE for prevalent 31/33/45 infection associated with 1, 2 and 3 vaccine doses was $-1.62 \%$ (95\% CI $-85.1,45.3), 48.3 \%$ (95\% CI 7.6, 71.8) and 55.2\% (95\% CI 32.6, 70.2). Thus, we observed an impact of one dose on the prevalence of vaccine-type infection. Furthermore, cross protection for HPV 31/33/45 with two doses was similar to that conferred by three doses even though the two doses were largely administered at 0 and 1 months (compared with three-dose schedule of 0,1 and 6 months).

\section{DISCUSSION}

Through the assessment of HPV infection in women immunised via a national catch-up programme, we observed an impact of 1 and 2 doses of vaccine on the prevalence of HPV 16/18 infectionalbeit lower than that associated with three doses. Notably, cross protection for HPV 31/33/45 with two doses was similar to that conferred by three doses even though the two doses were largely administered at 0 and 1 months. Vaccination with one dose did not confer a cross-protective effect against HPV 31/33/45.

There are caveats to our analysis; as immunisation uptake for the complete schedule has been consistently high in Scotland, relatively few women had $<3$ doses (http://www.isdscotland.org/ Health-Topics/Child-Health/publications/index.asp.) Consequently, rare outcomes such as mono infection with HPV 31, 33 and 45 are challenging to measure with current numbers. Furthermore, we did not have knowledge of HPV status before vaccination and outcomes could not be measured as incident or persistent infection. This is apposite considering that analysis was based on women vaccinated as part of a catch-up programme, so a proportion will have been exposed to HPV, pre-vaccination. In a previous HPV prevalence study in Scotland undertaken before the national vaccination programme, which utilised urine, $12.6 \%$ of women aged 15-18 were HR-HPV positive (O'Leary et al, 2011). Consequently, the VE described above is likely to be an underestimate of that expected in HPV-naïve women. In addition we also compared older unvaccinated women with younger vaccinated women, who may have different attitudes and behaviours with respect to health care/screening. In a recent observational study by Palmer et al (2016b), uptake of first invitation to cervical screening by vaccine status was assessed; immunised women in the catch-up cohorts showed higher attendence than unimmunised women, but this may be a result of a greater awareness of health issues and may not be replicated in routinely immunised women. Finally, it is feasible that a herd effect may have a bearing on what we have attributed to $<3$ doses.

It is of interest that our overarching observations are consistent with those of Kreimer et al, in spite of the clear differences between settings. Notably, the cross-protective data align more with those of the PATRICIA trial, rather than the combined (PATRICIA plus Costa Rica Vaccine trial) analysis, which reported a higher onedose VE, albeit a non-significant one. This may reflect differences in the individual contribution of HPV 31, 33 and 45 across settings, between-study HPV assay differences or, as indicated earlier, it may simply be an issue of power for this low-frequency outcome.

While an impact of one dose was observed, it was lower than that observed with three doses. As the bulk of two-dose data was associated with 0 and 1 month scheduling, we cannot compare it to 'extended' two-dose schedules, which is current policy in Scotland and other settings. The lower effectiveness of one dose, may be as a consequence of lower immunogenicity. In a recent study by LaMontagne et al (2014) of 376 Ugandan girls aged between 10 and 11 who received 1,2 and 3 doses of the bivalent vaccine, geometric mean antibody levels (GMTs) measured at 24 months for HPV 16/18 were inferior in women who received one dose compared with three doses. However, the authors noted that levels were still higher than those observed in adult women who received one dose in whom efficacy (for infection endpoints) had been demonstrated (LaMontagne et al, 2014). The authors also contended that the GMTs associated with one dose were higher than those generated as a consequence of natural infection where a partially protective effect had been demonstrated (Safaeian et al, 2010).

While we did/could not measure GMTs in the current analysis, given the average age of vaccination was much higher than the Ugandan study, it is tempting to speculate that one-dose VE will be higher in the routine (12-13 year old) cohorts given their lower likelihood of being infected before vaccination and an anticipated higher immunogenic response to the vaccine. It will be of interest to see how the VE estimates change when women who were vaccinated aged 12-13 enter the Scottish screening programme in late 2015.

To conclude, while we do not assert that current data on onedose protection are sufficient to influence policy, given that an 
impact has been observed in trial settings and in this and other population-based settings (Brotherton et al, 2015) there is now clear evidence to justify further, comprehensive assessment of onedose efficacy, given the global implications.

\section{ACKNOWLEDGEMENTS}

We thank all members of the Scottish HPV Reference Laboratory and the HPV Research group for support with sample processing and testing. We also thank NRS Lothian Bioresource (formerly SAHSC Bioresource) for support with sample capture and governance.

\section{CONFLICT OF INTEREST}

The authors declare no conflict of interest.

\section{REFERENCES}

Bhatia R, Kavanagh K, Cubie H, Serrano I, Wennington H, Hopkins M, Pan J, Pollock KG, Palmer TJ, Cuschieri K (2016) Use of HPV testing for cervical screening in vaccinated women-insights from the SHEVa (Scottish HPV Prevalence in Vaccinated Women) study. Int J Cancer; e-pub ahead of print 4 Feb 2016; doi:10.1002/ijc.30030.

Brotherton JM, Malloy M, Budd AC, Saville M, Drennan KT, Gertig DM (2015) Effectiveness of less than three doses of quadrivalent human papillomavirus vaccine against cervical intraepithelial neoplasia when administered using a standard dose spacing schedule: Observational cohort of young women in Australia. Papillomavirus Res 1: 59-73.

Drolet M, Bénard É, Boily MC, Ali H, Baandrup L, Bauer H, Beddows S, Brisson J, Brotherton JM, Cummings T, Donovan B, Fairley CK, Flagg EW, Johnson AM, Kahn JA, Kavanagh K, Kjaer SK, Kliewer EV, LemieuxMellouki P, Markowitz L, Mboup A, Mesher D, Niccolai L, Oliphant J, Pollock KG, Soldan K, Sonnenberg P, Tabrizi SN, Tanton C, Brisson M (2015) Population-level impact and herd effects following human papillomavirus vaccination programmes: a systematic review and meta-analysis. Lancet Infect Dis 15: 565-580.

Kavanagh K, Pollock KG, Potts A, Love J, Cuschieri K, Cubie H, Robertson C, Donaghy M (2014) Introduction and sustained high coverage of the HPV bivalent vaccine leads to a reduction in prevalence of HPV 16/18 and closely related HPV types. Br J Cancer 110: 2804-2811.

Kavanagh K, Sinka K, Cuschieri K, Love J, Potts A, Pollock KG, Cubie H, Donaghy M, Robertson C (2013) Estimation of HPV prevalence in young women in Scotland; monitoring of future vaccine impact. BMC Infect Dis 13: 519.

Kreimer AR, Struyf F, Del Rosario-Raymundo MR, Hildesheim A, Skinner SR, Wacholder S, Garland SM, Herrero R, David MP, Wheeler CM. Costa Rica Vaccine Trial and PATRICIA study groups (2015) Efficacy of fewer than three doses of an HPV-16/18 AS04-adjuvanted vaccine: combined analysis of data from the Costa Rica Vaccine and PATRICIA trials. Lancet Oncol 16: $775-786$.

LaMontagne DS, Mugisha E, Pan Y, Kumakech E, Ssemaganda A, Kemp TJ, Cover J, Pinto LA, Safaeian M (2014) Immunogenicity of bivalent HPV vaccine among partially vaccinated young adolescent girls in Uganda. Vaccine 32: 6303-6311.

Lowy DR, Herrero R, Hildesheim A. Participants in the IARC/NCI workshop on Primary Endpoints for Prophylactic HPV Vaccine Trials (2015) Primary endpoints for future prophylactic human papillomavirus vaccine trials: towards infection and immunobridging. Lancet Oncol 16: e226-e233.

O’Leary MC, Sinka K, Robertson C, Cuschieri K, Lyman R, Lacey M, Potts A, Cubie HA, Donaghy M (2011) HPV type-specific prevalence using a urine assay inunvaccinated male and female 11- to 18-year olds in Scotland. Br J Cancer 104: 1221-1226.

Palmer TJ, McFadden M, Pollock KG, Kavanagh K, Cuschieri K, Cruickshank M, Cotton S, Nicoll S, Robertson C (2016a) HPV immunisation and cervical screening-confirmation of changed performance of cytology as a screening test in immunised women: a retrospective population-based cohort study. Br J Cancer 114: 582-589.

Palmer TJ, McFadden M, Pollock KG, Kavanagh K, Cuschieri K, Cruickshank M, Nicoll S, Robertson C (2016b) HPV immunisation and increased uptake of cervical screening in Scottish women; observational study of routinely collected national data. Br J Cancer 114: 576-581.

Pollock KG, Kavanagh K, Potts A, Love J, Cuschieri K, Cubie H, Robertson C, Cruickshank M, Palmer TJ, Nicoll S, Donaghy M (2014) Reduction of low- and high-grade cervical abnormalities associated with high uptake of the HPV bivalent vaccine in Scotland. Br J Cancer 111: 1824-1830.

Safaeian M, Porras C, Schiffman M, Rodriguez AC, Wacholder S, Gonzalez P, Quint W, van Doorn LJ, Sherman ME, Xhenseval V, Herrero R, Hildesheim A. Costa Rican Vaccine Trial Group (2010) Epidemiological study of anti-HPV16/18 seropositivity and subsequent risk of HPV16 and -18 infections. J Natl Cancer Inst 102: 1653-1662. 